\title{
Review of Relief Demand Forecasting Problem in Emergency Logistic System
}

\author{
Jianan Zhao, Cejun Cao* \\ School of Management, Jinan University, Guangzhou, China \\ Email: "caocejun0601@126.com
}

Received 16 January 2015; accepted 3 February 2015; published 9 February 2015

Copyright (C) 2015 by authors and Scientific Research Publishing Inc.

This work is licensed under the Creative Commons Attribution International License (CC BY).

http://creativecommons.org/licenses/by/4.0/

(c) (i) Open Access

\begin{abstract}
Demand forecasting on relief is the premise and basis of material allocation scheme in emergency logistic system. Reasonable demand forecasting method can facilitate relief distribution, thus avoiding the phenomenon that supply-demand imbalance and relief distribution delay. In this paper, relief will be categorized from point view of government and academia, to explain the relationship between relief categorization and demand forecasting. Then introduce the characteristics of relief-demand from several aspects, such as sudden, uncertainty, timeliness, and stage. Finally, this paper gives an overall conclusion on current development of relief demand forecasting method. And elaborate the application of case-based reasoning, information entropy theory, considering safety stock in the field of relief-demand forecasting in detail, to provide reference for relief distribution.
\end{abstract}

Keywords

Relief, Material Categorization, Demand Characteristic, Forecasting Method, Review

\section{Introduction}

In the past few decades, it results in a worse negative effect on society and economy with the frequency of emergencies increasing. For example, Sarin gas attacked on Tokyo subway in 1995. Severe Acute Respiratory Syndromes (SARS) as an epidemic disease spread in 2003 in China. The southern big snowstorm and Wenchuan earthquake happened in 2008 in China. In addition, the earthquake occurred in Yaan and Chile, and so on. All these emergencies lead to heavy loss of human lives and properties [1]. That needs a large quantity of reliefs in order to reduce or relieve this loss. And the sudden of emergency shows that it is difficult to predict or the interval time between forecasting and occurrence is extremely short. What described above all puts forward new challenges for emergency logistics system. Therefore, this paper focusing on emergency logistic system has certain practical significance.

"Corresponding author. 
Emergency logistic system discussed here refers that, in emergency situation, to meet (or finish) the urgent (or sudden) logistic demand of the victims, it is the process of effective planning, managing, and controlling of reliefs, information and rescue service from supply point to demand point. To reduce or relieve the serious results of emergencies, improving the accuracy and reliability of relief demand forecasting is an effective solution. By analyzing the relief types and characteristics of relief demand, it shows that a reasonable and proper forecasting method can make for distributing relief efficiently and quickly as well as avoiding the phenomenon that supplydemand imbalance and relief distribution delay. Therefore, the study on relief demand forecasting problem in emergency logistic system has a certain practical and theoretical significance.

In comparison, scholar home and broad mainly focused on the relief transportation and distribution in emergency logistic system [2]. And the relief demand amount to affected areas is known in these literatures. It is treated as a prerequisite. However, during the emergency response process in reality, it is difficult to obtain the information (e.g. quantity, type, demand structure, etc.) about relief demand because of being limited by various objective conditions. Recent years, aiming at this problem, scholar applied various theories, methods and techniques to predict the relief demand amount each affected area in emergency logistic system. Meanwhile, there are a lot of achievements. These methods can be classified into two groups, namely quantitative and qualitative method. Qualitative method or theory includes Fuzzy Comprehensive Evaluation (FCE) [1], Analytic Hierarchy Process (AHP), and Delphi. Quantitative method or theory includes time-series [3] [4], comprehensive analysis [5], case-based reasoning [6]-[9], information entropy [10], and safety stock [11], and so on. Above all, the techniques or technologies of relief-demand forecasting develop from qualitative analysis completely to combining qualitative with quantitative method, and from linear statistical analysis to nonlinear artificial intelligence method.

In this paper, we categorize relief from point view of government and academia, and then explain the relationship between relief categorization and demand forecasting. Then the characteristics of relief-demand, such as sudden, uncertainty, timeliness, and stage are introduced. Finally, an overall conclusion on the current development of relief demand forecasting method is given.

\section{Relief Classification in Emergency Logistic System}

Relief discussed here refers to all types of material needed during emergency response process. It is necessary to classify all relief in order to enhance the efficiency of scheduling and the forecasting accuracy of relief demand. Relief classification enhances emergency preparedness and readiness by the whole community through a system that allows jurisdictions to augment their capabilities during an incident.

\subsection{Relief Classification from Point View of Government}

There are many classification methods on relief home and abroad. For instance, Federal Emergency Management Agency (FEMA) mentioned that relief can be classified into nine categories, namely emergency medical services resources, incident management resources, medical and public health resources, law enforcement resources, public works resources, search and rescue resources, mass care resources, fire and hazardous materials resources, animal emergency response resources respectively. And each category is compiled manual separately. In Japan, according to the application of relief, disaster emergency material includes three categories, namely foods, daily articles and self-help tools and so on. Australian Federal Government Emergency Management Agency divides relief into physical resources and technical resources in Overseas Disaster Rescue Plan. And resource in emergency management arrangement includes search and rescue resources, medical aid resources, foods and others.

Emergency support material classification and production list made by Ministry of Civil Affairs of the People's Republic of China shows that relief can be divided into thirteen categories, namely protective equipment resources, life-saving resources, life support resources, load and transport resources for rescue, temporary accommodation resources, pollution and clean-up resources, power fuels resources, engineering equipment resources, engineering materials resources, equipment tools resources, lighting equipment resources, radio communication resources and transport resources.

\subsection{Relief Classification from Point View of Academia}

In literature [12], professor Fiedrich from Karlsruhe University of Germany treated initial or key rescue stage in 
earthquake disaster as the object. And reliefs are defined as the machines and equipment which can be used to simultaneously work at different affected areas. In detail, they include six categories, namely search-and-rescue group (to accomplish rescue tasks), crane (to accomplish lift tasks), hydraulic excavator (to use for lift and load tasks), wheeled loader (to use for load tasks), truck (to accomplish transport tasks), and roller (to use for compact tasks). However, this literature only focuses on different equipment used in search and rescue areas, stabilizing areas, and rehabilitation areas. And it belongs to similar resource allocation problem.

In literature [10] [11], for emergency logistic distribution problem for quick response to urgent relief demand in earthquake, professor Sheu from National Chiao Tung University in Taiwan consider two types of urgent relief needed in affected areas including daily-used equipment for refugees and daily consuming relief. What's more, the former mainly refers to water and meal boxes, and the latter refers to sleeping bags and camps.

In literature [13], based on the characteristics of rescue operational process in flood disaster management, Qin from Xi'an Jiaotong University in China mentioned that these necessary emergency materials can be classified into two categories, namely response resources (e.g. sandbags and protection network) and recovery resources (e.g. food and medicine). During the process of emergency handling, the relationship between the two reliefs is independent. However, due to complexity and serious degree of this problem, this relationship is non-linear and stochastic. In addition, some scholar divided the relief needed in emergency management of drought disaster into pumping equipment and irrigation equipment.

According to the detail description above all, we can conclude that the type of emergency relief is various. The forecasting methods of different types of relief are various (or make a distinction between these methods). So relief classification is the prerequisite and basis in terms of relief-demand amount forecast in emergency logistic system. During emergency response process, only to master the relief classification related information clearly, the amount of relief-demand can be predicted exactly.

\section{Relief Demand Forecasting in Emergency Logistic System}

\subsection{The Characteristics of Relief Demand}

After emergency, the relief demand in the affected areas has four features, namely sudden, uncertainty, timeliness, stage.

\section{1) The sudden of relief-demand}

The sudden is the typical characteristic of emergency. This sudden reflects not only the occurrence of emergency by accident but also the change process of disaster elements from quantity to quality. It is this sudden of emergency that leads to the sudden of relief-demand.

\section{2) The uncertainty of relief-demand}

This characteristic is relatively obvious by comparing with the traditional material demand. With the development of emergency response process, the relief demand in the affected area is varied dynamically. The consequences or phenomenon of emergency, such as serious damage of infrastructure, the blocking of communication, and the block of transportation, results in some information being unable to obtain in time. This information involve in the total number of fatalities, the population consistency, and the degree of damage in the affected area. The unsymmetrical phenomenon of information occurs between the victims and decision-makers. Thus the relief demand on amount and type put up uncertainty.

\section{3) The timeliness of relief-demand}

Time is the key factor needed to be considered into the relief allocation problem during emergency response process. Relief transportation from supply point to demand site or the affected area should be accomplished as soon as possible. That is the primary task during emergency response process. Time is limited for decisionmaker after emergency. It is necessary to make the relief allocation scheme in time for decision-maker instead of missing the best chance. In addition, the timeliness of relief arriving at the affected area has a direct effect on the damage range of emergency. To a certain extent, that reliefs arrive in time can reduces the occurrence frequency of sub-emergency or secondary emergency. Thus it can relive the loss brought by emergency.

\section{4) The stage of relief-demand}

Emergency response process can be refined three stages in terms of golden rescue stage, buffer rescue stage, and the recovery stage [14]. Different stages have different relief-demand structure. For example, the large rescue equipment and medicine articles are rare during golden rescue stage. In buffer rescue stage, the quantity of demand on tents and foods has a more large scale. Analyze the characteristics of relief-demand properly, that is 
good to identify the focused or key problem during the process of designing relief demand forecasting method in emergency logistic system.

\subsection{Relief Demand Forecasting Method in Emergency Logistic System}

The process of relief allocation in emergency logistic system involves in four parts including relief-demand forecasting, groping the affected area, classification of the victims, and relief distribution. It is depicted in Figure 1 in detail.

According to Figure 1, we can find that relief demand forecasting in the affected area plays an important role in the process of distribution. The rescue effects directly depend on the accuracy of relief-demand forecasting. Meanwhile the selection of forecasting method is the key problem. Recently, there are a lot of researches in the field of relief-demand forecasting method in emergency logistic system. Also there are more fruitful achievements. In this paper, on the basis of relief classification, by analyzing the characteristics of relief-demand we make a conclusion about relief demand forecasting method in emergency logistic system in terms of quantitative method and qualitative method.

\subsubsection{The Qualitative Methods of Relief Demand Forecasting}

At the beginning of emergency management, traditional relief-demand forecasting of the affected area mainly depends on decision-makers' experience and experts' judgment subjected to various limitation constraints. Expert scoring method has been applied widely into relief-demand forecasting including Delphi, Fuzzy Comprehensive Evaluation (FCE), and Analytic Hierarchy Process (AHP). To a large extent, Expert scoring method is suitable for complex relief-demand forecasting. But this method is too subjective to explain. Above all, the application of this method in relief-demand forecasting of real emergency is limited seriously. For instance, Sun [1] proposed a fuzzy rough set approach to emergency material demand prediction over two universes.

\subsubsection{The Quantitative Methods of Relief Demand Forecasting}

The qualitative method can't well adapt to emergency management over time. Based on qualitative analysis, scholar introduced various quantitative methods to predict the relief-demand amount in emergency logistic system. With the development of emergency management and statistics, scholars begin to apply some statistics analysis method to the field of relief-demand forecasting. They are mainly time-series forecasting methods (e.g. Autoregressive and Integrated Moving Average-ARIMA, exponential smoothing models, and independent identically distribution models) and comprehensive analysis methods. However, the amount of relief-demand after emergency usually shows a great deal of non-linear and non-regulatory, to result in forecasting of traditional statistic method being ineffective. What's more, the quantitative methods include still artificial intelligence model (e.g. Neural Networks), case-based reasoning, information entropy theory, and safety stock modelling. The following section will concentrate on the latter three methods' application in the field of relief-demand forecasting in emergency logistic system.

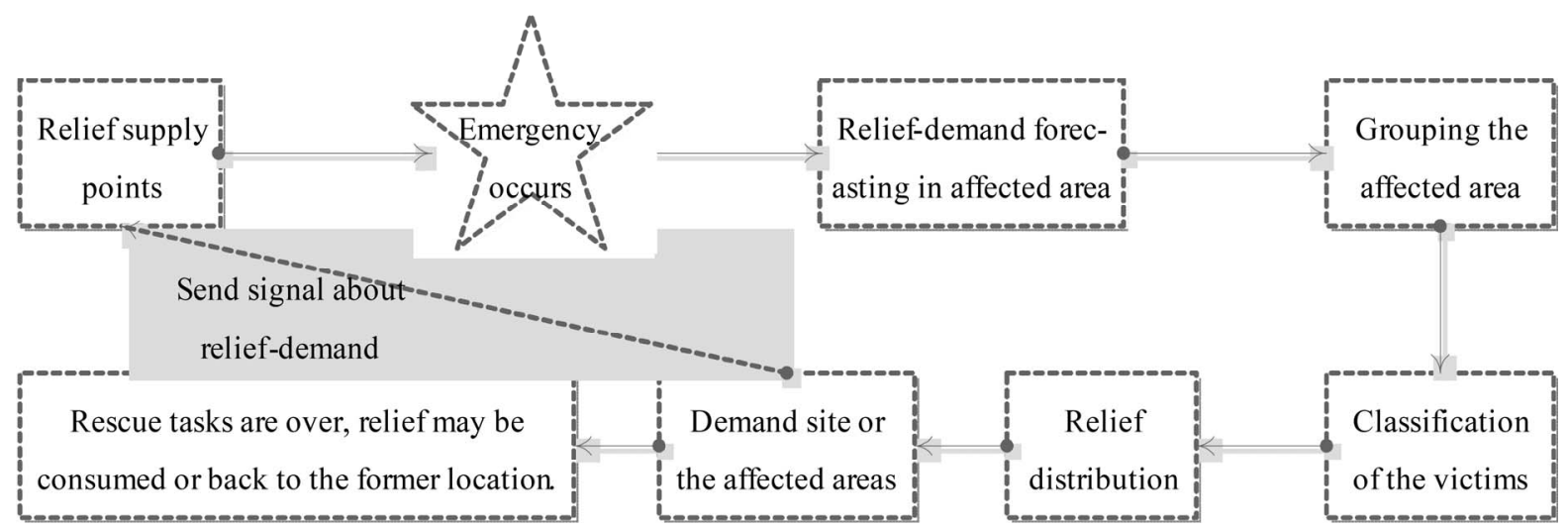

Figure 1. The relief distribution process of emergency logistic system. Data source: finished based on literature [10]-[11] [14] by authors. 


\section{1) Relief demand forecasting based on case-based reasoning}

Case-based Reasoning (CBR) method is proposed in dynamic memory written by Roger in 1982. And it is applied and verified gradually by his students over time. Recent years, scholars began to apply CBR method into relief-demand forecasting. In detail, it involves unconventional emergency, water transport emergency, and public emergency. The representatives include Professor Liu of Tsinghua University, Professor Liu Mao of Nankai University, Professor Zhuang Yaming of Southeast University, and Deng Shoucheng engineer. All in all, reliefdemand forecasting based on CBR is composed of retrieve, reuse, revise and retain [15], which is depicted in Figure 2.

In detail, the process of CBR can be described as follows. Analyze the influencing factors of relief-demand based on relief classification by combining with the characteristics of relief-demand forecasting process. And then abstract the features of indexes, determine the weight of index, calculate the similarity between target case and source case based on formula (1), and obtain the amount of relief in target case or emergency.

$$
\operatorname{Sim}\left(T, C_{i}\right)=\frac{\sum_{j=1}^{m} w_{j}\left(n_{T}\left(x_{j}\right) \wedge n_{C_{i}}\left(x_{j}\right)\right)}{\sum_{j=1}^{m} w_{j}\left(n_{T}\left(x_{j}\right) \vee n_{C_{i}}\left(x_{j}\right)\right)} \geq \eta, i=1,2, \cdots, \mathrm{n}
$$

And in formula (1), the target case is denoted by $T$, the source case is denoted by $C_{i}$, and the similarity between target case and source case is denoted by $\operatorname{Sim}(T, C)$. Relief-demand forecasting based on Case-based Reasoning method needn't depend on infinite model. It improves the efficiency of solving problem by reusing the past solutions. At the same time, it can retain learning continuously by acquiring new knowledge from the cases.

\section{2) Relief demand forecasting based on information entropy theory}

Entropy theory was put forward by Rudolf Clausius, and first applying to thermodynamics field. With the development of this theory, its applications are more widely. Up to 1948, Shannon as the first scholar introduced entropy into information field. Professor Sheu [10] assumed that the time-varying relief demand needed in a given affected area is highly correlated with the number of survivals. And based on this prerequisite, literature [10] studied daily-consuming relief, such as water, meal boxes and so on, distribution problem. Then propose and entropy-based weighting technique to fuse multi-source fatality data in real time, calculate the number of fatality in the affected area at current time, and predict the number of relief-demand. Its expression can be denoted by formula (2).

$$
D_{i}(t)=\alpha \times S_{i}(t) \times \bar{L}+z_{1-\alpha} \times V A R_{i}(t) \times \sqrt{\bar{L}}
$$

And in formula (2), $D_{\mathrm{i}}(t)$ represents the instantaneous relief-demand of affected area. $a$ is a parameter representing the average hourly relief demand needed per survival in the affected area. $\bar{L}$ is the upper bound of

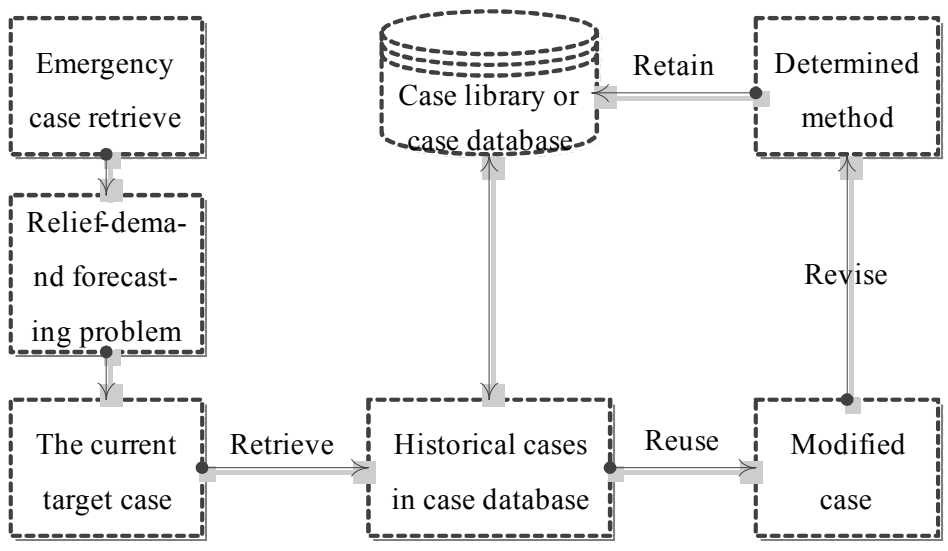

Figure 2. Process of relief-demand forecasting based on CBR. Data source: finished based on literature [15] by authors 
the tolerable lead time for relief distribution to any given affected area. And the parameter $\alpha$ should meet the equation as follows.

$$
\operatorname{Prob}\left(\bar{L} \leq D_{\mathrm{i}}(t)\right)=1-\alpha
$$

In addition, $S_{\mathrm{i}}(t)$ represents the instantaneous amount of relief-demand needed by the trapped survivals in affected area. It can be noted by:

$$
S_{i}(t)=\delta_{\mathrm{i}}-X_{\mathrm{i}}(t)
$$

where, $\delta_{i}$ represents the existing population of affected area which is assumed to be available in advance from the socio-economic database of the corresponding local government. The accumulated number of fatalities associated with a given affected area, being denoted by $X_{\mathrm{i}}(t)$. And this variable is related with the uncertainty level of information source in the given affected area. That is to say, $X_{\mathrm{i}}(t)$ is related with $H(t)$ representing information entropy. It is can be denoted by:

$$
H(t)=-\sum_{\mathrm{m}=1}^{\mathrm{M}} p(m \mid x(t)) \log [p(m \mid x(t))]
$$

And, $\operatorname{VAR}_{\mathrm{i}}(t)$ represents the temporal variability of relief demand, which is given by:

$$
\operatorname{VAR}_{i}(t)=\sqrt{\frac{\sum_{\varepsilon=0}^{t-1}\left[D_{i}(t-\varepsilon)-\bar{D}_{\mathrm{i}}(t)\right]^{2}}{t}}
$$

\section{3) Relief demand forecasting based on safety stock theory}

Professor Sheu from National Chiao Tung University in Taiwan [11] assumed that the time-varying relief demand needed in a given affected area is highly correlated with the number of corresponding local survivals, being similarity with literature [10]. This paper treated daily consuming relief and daily-used equipment as the object. And according the characteristics of the reliefs, give respectively the relief-demand forecasting method after emergency. Thus in terms of daily consuming relief, the demand amount includes two parts with safety stock and the real demand of survivals, which is given by:

$$
D_{i}(t)=\max \left\{a \times \delta_{i}(t) \times \bar{L}+z_{1-a} \times S T D_{i}(t) \times \sqrt{\bar{L}}, 0\right\}
$$

And, in terms of daily-used equipment, the demand amount of this relief considers three parts, which can be denoted by:

$$
D_{i}(t)=\max \left\{a \times \delta_{i}(t)+b_{i}-C, 0\right\}
$$

For formula (7) and (8), parameter $a, \bar{L}$ and $\alpha$ has the same meaning with formula (2). And $\delta_{i}(t)$ represents the estimated number of survivals trapped in an affected area in a given time interval $t$. The corresponding buffer demand or safety stock associated with this relief is denoted by $b_{i}$. And $C$ represents the accumulated time-varying amount of relief arriving at the affected area in a given time interval $t$. The time-varying standard deviation of relief demand associated with the affected area is denoted by $\operatorname{STD}_{\mathrm{i}}(t)$.

All kinds of methods of relief-demand forecasting mentioned above all were applied more or less to the process of relief distribution in some real cases, such as the massive earthquake measured 7.3 on the Richter scale, which occurred in central Taiwan, The southern big snowstorm and Wenchuan earthquake happened in 2008 in China, and 8.2 magnitude earthquake occurred in Chile in 2014, and so on.

\section{Conclusions}

For a variety of emergencies, the phenomenon that supply-demand imbalance and relief distribution delay in emergency logistic system has been attracted more attention by people from all walks of life. For this problem, enhancing the relief-demand forecasting accuracy is an effective way. Fundamentally, the forecasting accuracy is highly correlated with relief classification, the characteristics of relief-demand in the affected area, and the selection of relief-demand forecasting method. 
In this paper, we analyze and summarize relief-demand forecasting problem in emergency logistic system based on the main line that relief classification - the analysis of demand characteristics - relief demand forecasting method. This paper makes a conclusion as follows: 1) Identifying the relief classification and the characteristics of relief-demand in the affected area is the basis and prerequisite of relief-demand forecasting in emergency logistic system. 2) The proper and reasonable forecasting method selected is an effective way of avoiding the phenomenon that supply-demand imbalance and relief distribution delay. 3) The relief-demand forecasting demand method develops from based on experience or expert judgment towards quantitative, information technology and intelligent. Above all, during the era of rapid development of information technology, cloud computing and big data bring relief-demand forecasting with not only challenges but also opportunities.

\section{Acknowledgements}

It is warmly appreciated the whole participated reviewers. And this paper is financially supported by the projects including 2014 Jinan University scientific research creativeness project for outstanding graduate and 2014 Jinan University Challenge Cup student extracurricular scientific entrepreneurship contest.

\section{References}

[1] Sun, B.Z., Ma, W.M. and Zhao, H.Y. (2013) A Fuzzy Rough Set Approach to Emergency Material Demand Prediction over Two Universes. Applied Mathematical Modelling, 37, 7062-7070. http://dx.doi.org/10.1016/j.apm.2013.02.008

[2] Wilson, D.T., Hawe, G.I., Coates, G. and Crouch, R.S. (2013) A Multi-Objective Combinatorial Model of Casualty Processing in Major Incident Response. European Journal of Operational Research, 230, 643-655. http://dx.doi.org/10.1016/j.ejor.2013.04.040

[3] Xu, X.Y., Qi, Y.Q. and Hua, Z.S. (2010) Forecasting Demand of Commodities after Natural Disasters. Expert Systems with Applications, 37, 4313-4317. http://dx.doi.org/10.1016/j.eswa.2009.11.069

[4] Jones, S.S., Evans, R.S., Allen, T.L., et al. (2009) A Multivariate Time Series Approach to Modeling and Forecasting Demand in the Emergency Department. Journal of Biomedical Informatics, 42, 123-139. http://dx.doi.org/10.1016/j.jbi.2008.05.003

[5] Ninno, C.D., Dorosh, P.A. and Smith, L.C. (2003) Public Policy, Markets and Household Coping Strategies in Bangladesh: Avoiding a Food Security Crisis Following the 1998 Flood. World Development, 31, 1221-1238. http://dx.doi.org/10.1016/S0305-750X(03)00071-8

[6] Liu, W.M., Hu, G.Y. and Li, J.F. (2012) Emergency Resources Demand Prediction Using Case-Based Reasoning. Safety Science, 50, 530-534. http://dx.doi.org/10.1016/j.ssci.2011.11.007

[7] Wang, W. and Liu, M. (2010) Method of Emergency Resources Demand Forecasting Based on Case-Based Reasoning. Journal of Safety and Environment, 10, 217-220.

[8] Deng, S.C., Wu, Q., Shi, B., et al. (2014) Prediction of Resource for Responding Waterway Transportation Emergency Based on Case-Based Reasoning. China Safety Science Journal, 24, 79-84.

[9] Wang, X. and Zhuang, Y. (2010) Forecasting Model of Unconventional Emergence Incident's Resource Demand Based on Case-Based Reasoning. Journal of Xidian University (Social Science Edition), 20, 22-26.

[10] Sheu, J.B. (2010) Dynamic Relief-Demand Management for Emergency Logistics Operations under Large-Scale Disasters. Transportation Research Part E: Logistics and Transportation Reviews, 46, 1-17. http://dx.doi.org/10.1016/j.tre.2009.07.005

[11] Sheu, J.B. (2007) An Emergency Logistics Distribution Approach for in Disasters. Transportation Research Part E: Logistics and Transportation Reviews, 43, 687-709. http://dx.doi.org/10.1016/j.tre.2006.04.004

[12] Fiedrich, F., Gehbauer, F. and Rickers, U. (2000) Optimized Resource Allocation for Emergency Response after Earthquake Disasters. Safety Science, 35, 41-57.

[13] Qin, J.C., Xing, Y.T., Wang, S., et al. (2012) An Inter-Temporal Resource Emergency Management Model. Computers \& Operations Research, 39, 1909-1918. http://dx.doi.org/10.1016/j.cor.2011.07.008

[14] Li, C.D., Cao, C.J. Yang, Q., et al. (2014) Application of BOX theory in Multi-Stage Emergency Resource SchedulingTaking Emergency Response Stage as an Example. China Safety Science Journal, 24, 159-165.

[15] Roger, C.S. and David, B.L. (1989) Creativity and Learning in a Case-Based Explainer. Artificial Intelligence, 40, 353-385. http://dx.doi.org/10.1016/0004-3702(89)90053-2 